\title{
Analysis of Offshore Knuckle Boom Crane - Part One: Modeling and Parameter Identification
}

\author{
Morten K. Bak Michael R. Hansen
}

Department of Engineering Sciences, Faculty of Engineering and Science University of Agder, 4879 Grimstad, Norway. E-mail: \{morten.k.bak,michael.r.hansen\} @uia.no

\begin{abstract}
This paper presents an extensive model of a knuckle boom crane used for pipe handling on offshore drilling rigs. The mechanical system is modeled as a multi-body system and includes the structural flexibility and damping. The motion control system model includes the main components of the crane's electro-hydraulic actuation system. For this a novel black-box model for counterbalance valves is presented, which uses two different pressure ratios to compute the flow through the valve. Experimental data and parameter identification, based on both numerical optimization and manual tuning, are used to verify the crane model.

The demonstrated modeling and parameter identification techniques target the system engineer and takes into account the limited access to component data normally encountered by engineers working with design of hydraulic systems.
\end{abstract}

Keywords: Hydraulic crane, multi-body system, flexibility, directional control valve, counterbalance valve

\section{Introduction}

Today's offshore drilling equipment is characterized by high price, high level of system complexity and low production numbers. For the equipment manufacturers, it requires a great level of skill and experience to develop the equipment, since there are very limited possibilities to build prototypes for testing and verification of new designs. Increasing focus on production and development costs adds to this challenge. As a consequence, design engineers continuously have to improve their procedures for decision making regarding choice of principal solutions, components and materials in order to reach the best possible trade-off between different performance criteria such as reliability, efficiency and cost.

Computer based time domain simulation and optimization techniques have, by far, proven themselves as excellent tools for the challenged designer and have over the last couple of decades increasingly been em- ployed by drilling equipment manufacturers. However, the use of these techniques still offers a number of challenges both in industry as well as academia.

In model based design, simulation models serve as virtual prototypes providing information, e.g., about a machine's overall efficiency, stability and accuracy, enabling engineers to test, redesign and optimize the design of the machine before it is manufactured. Model based design offers the possibility to reduce both development time and costs while also producing more reliable machines.

The main challenge in model based design lies within the ability to produce simulation models that, with a reasonable precision, are able to mimic the behavior of a real system. This challenge is especially pronounced for hydraulically actuated machines, like many offshore drilling applications, simply because suppliers of hydraulic component are not used to deliver all the data needed to develop simulation models of their products. 
An application that represents a typical piece of high-end offshore equipment is the knuckle boom crane. The ability to employ a model based approach for design of such cranes is highly relevant.

Modeling, simulation, design and control of various types of cranes have been subjected to extensive research. General modeling techniques and different control concepts have been presented by Hiller (1996) and Abdel-Rahman et al. (2003) and particularly mobile (truck-mounted) cranes have attracted a considerable amount of interest from researchers (Ellman et al., 1996), (Mikkola and Handroos, 1996), (Esque et al., 1999), (Hansen et al., 2001), (Nielsen et al., 2003) and (Esque et al., 2003). The dynamics of these types of cranes is well documented and modeling techniques have been proven through experimental verification.

In (Than et al., 2002) and (Bak et al., 2011) offshore boom cranes have been investigated. Models taking the structural flexibility into account have been presented, however, without any experimental verification.

Though mobile cranes are particularly flexible and behave differently than offshore cranes, the same modeling techniques can be used for both types of cranes. Modeling of mechanical systems such as boom mechanisms can be handled with different generic approaches. However, the most suitable approach is not always obvious.

Modeling approaches for hydraulic components and systems are, in general, also well-established but may cause problems when it comes to model verification. The problem is often a lack of proper model data and/or that the physics is not fully understood. Therefore, for certain hydraulic components, there may be a need to introduce new modeling approaches. In this paper an extensive model of an offshore knuckle boom crane is developed with a view to identify a best practice for predicting the behavior of this type of crane. It is demonstrated how to overcome the modeling challenge by choosing an appropriate level of modeling detail and by using experimental work together with parameter identification techniques.

A commercially available software package, MapleSim $^{\mathrm{TM}}$, is used to develop a dynamic model and MATLAB ${ }^{\circledR}$ is used for steady-state simulations and optimization based parameter identification.

\section{Considered System}

Knuckle boom cranes are used for a wide range of offshore and marine operations and therefore exist in different variations. The considered crane is manufactured by Aker Solutions and is used on drilling rigs to move drill pipes between the pipe deck and a transportation system leading to the drill floor of the rig. Prior to commissioning of a crane, it undergoes a test procedure to verify the functionality and ensure that the performance corresponds to the criteria given in the design specification. This procedure facilitates an experimental study that can be used to calibrate and verify design models of the crane. In the following a description of the considered crane is given along with system variables that have been measured and recorded during a test procedure. The procedure itself is described in section 5 along with the model parameter identification.

The considered crane may be treated as a large multidomain system consisting of three interacting systems:

1. A mechanical system.

2. An electro-hydraulic actuation system.

3. An electronic control system.

The main components of the cranes mechanical system are a rotating part mounted on a pedestal, an inner jib, an intermediate jib, an outer jib and a gripping yoke, see Fig. 1.

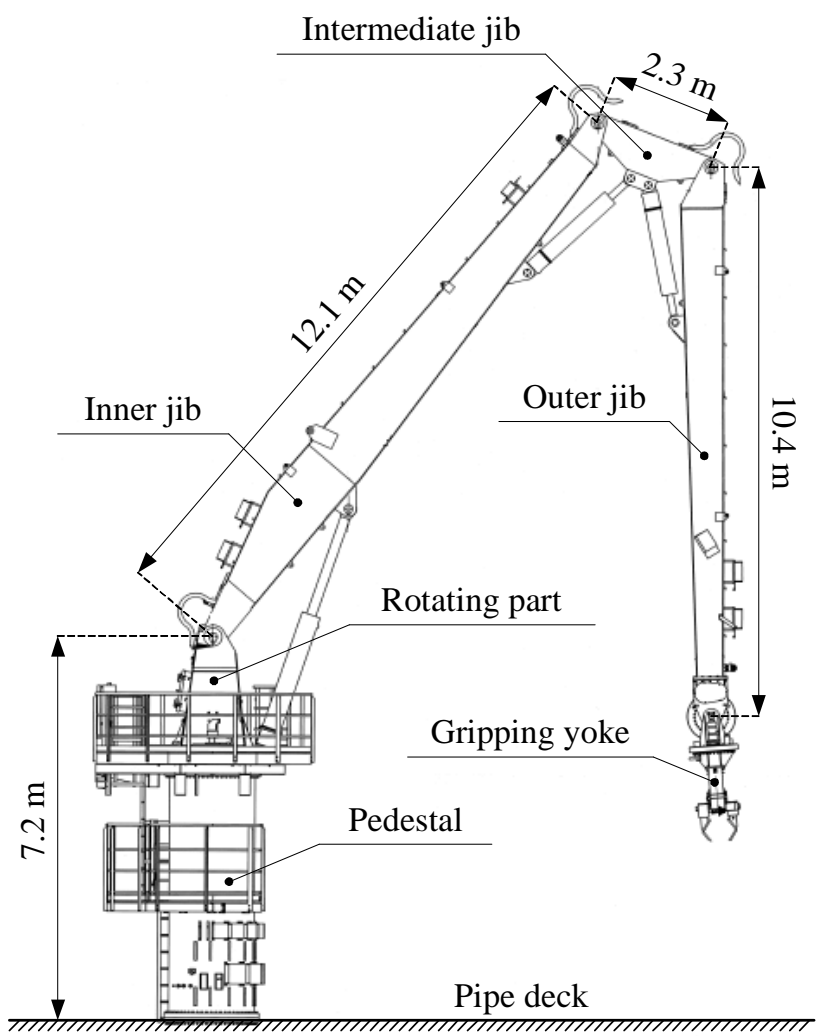

Figure 1: Main components of the mechanical system.

The crane is controlled from the operators cabin (not 
shown) mounted on the rotating part, also called the king. A slewing bearing and transmission between the king and the pedestal allows for slewing of the crane. However, this degree of freedom (DOF) and the details of the slewing transmission are not considered here.

The gripping yoke also includes a number of hydraulically actuated DOFs which are not considered. The actuation system is supplied by a hydraulic power unit (HPU) with constant supply pressure, $p_{S}=210$ bar, and return pressure, $p_{R}=0$.

The considered part of the actuation system consists of three hydraulic circuits, one for each crane jib, connected to the supply and return lines of the HPU. The control system includes a human-machine interface (HMI) which facilitates the operation of the crane, a number of sensors and instruments used for feedback control and/or monitoring and a controller where the control logic is defined.

When considering the actuation and control systems together, the three circuits of the actuation system can be considered as three sub-systems of the motion control system, including both actuation and control. A simplified schematic of the motion control sub-system for the inner jib is shown in Fig. 2.

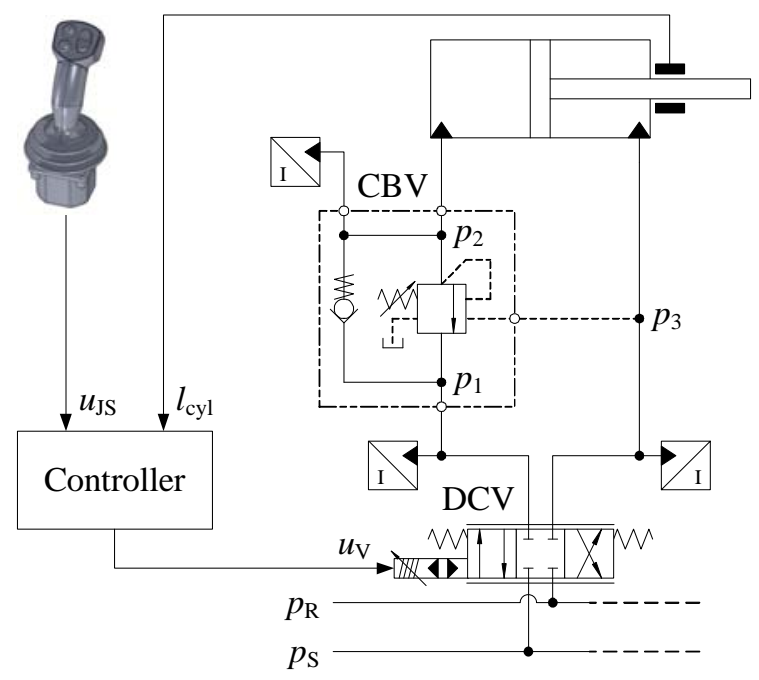

Figure 2: Simplified schematic of motion control subsystem for inner jib.
The main components of the hydraulic circuit are a cylinder with integrated position sensor, a servotype directional control valve (DCV) and an externally vented (drained) counterbalance valve (CBV). The cylinder velocity is controlled via the DCV, which controls the flow into either of the two cylinder chambers. During retraction of the cylinder (load lowering), it is exposed to negative loads (piston velocity and load force have the same direction) and therefore the piston pressure, $p_{2}$, needs to be controlled. This is handled by the $\mathrm{CBV}$, which provides a relief valve functionality on the outlet side assisted by the pressure, $p_{3}$, on the inlet side.

The DCV is controlled via a joystick (part of the HMI) from which the command signal, $u_{J S}$, is fed to the controller and used to generate the control signal, $u_{V}$, to the valve. The signal from the position sensor in the hydraulic cylinder is also fed to the controller, where it can be used for feedback control depending on the selected control mode. In open loop control mode joystick commands are passed through the controller and fed directly to the DCV. In closed loop control mode both joystick commands and cylinder positions are used for control of the DCV.

The motion control sub-systems for the intermediate and outer jibs are identical and contain the same control system elements as the one for the inner jib. However, as seen from Fig. 3, the elements of the actuation systems are different.

The DCV is pressure compensated and uses a load sensing (LS) circuit and a pressure reducing valve that maintains a constant pressure drop across metering edge of the main spool at any time. This makes the controlled flow independent of the load pressure and proportional to the spool position, i.e., the control signal fed to the valve.

There are two CBVs since the load force on the cylinder may act in either direction, depending on the orientation of the crane jibs. The CBVs are non-vented and include two series connected orifices to manipulate the pilot pressures, $p_{x, 1}$ and $p_{x, 2}$. For practical reasons it is only possible to measure $p_{2}$ and $p_{3}$ this circuits.

In total seven pressures, three command signals and three position signals are measured and recorded dur-

Table 1: System variables for considered motion control sub-systems.

\begin{tabular}{lcccccccc}
\hline$\checkmark=$ measured & Input & Output & \multicolumn{5}{c}{ State variables } \\
\cline { 2 - 9 } $\boldsymbol{x}=$ not measured & $u_{J S}$ & $l_{c y l}$ & $p_{1}$ & $p_{2}$ & $p_{3}$ & $p_{4}$ & $p_{x, 1}$ & $p_{x, 2}$ \\
\hline Inner jib & $\checkmark$ & $\checkmark$ & $\checkmark$ & $\checkmark$ & $\checkmark$ & NA & NA & NA \\
Intermediate jib & $\checkmark$ & $\checkmark$ & $x$ & $\checkmark$ & $\checkmark$ & $x$ & $x$ & $x$ \\
Outer jib & $\checkmark$ & $\checkmark$ & $x$ & $\checkmark$ & $\checkmark$ & $x$ & $x$ & $x$ \\
\hline
\end{tabular}




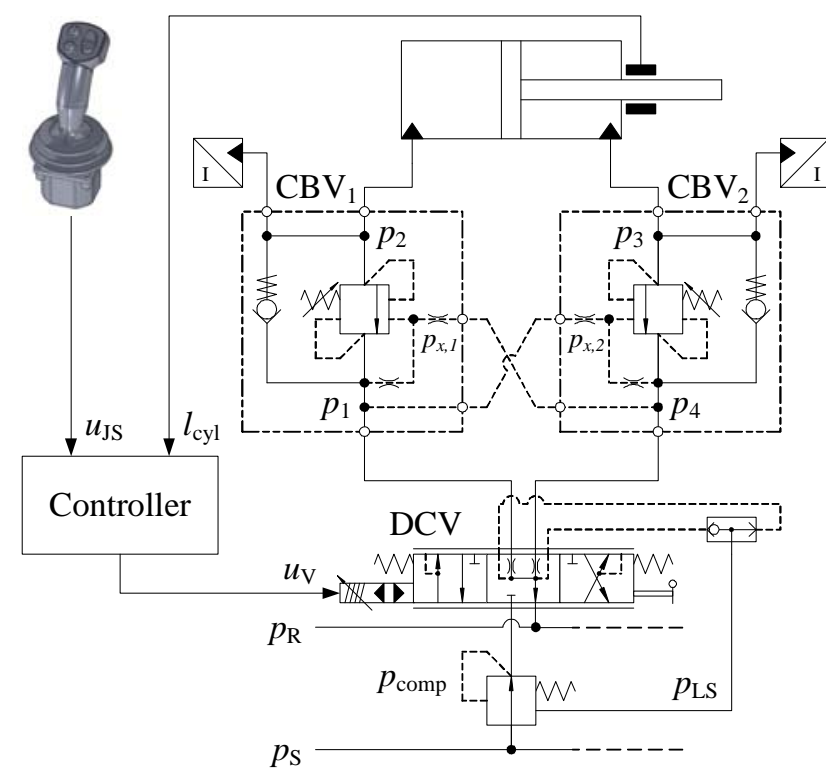

Figure 3: Simplified schematic of motion control subsystem for intermediate and outer jibs.

ing the experimental test procedure. Table 1 provides an overview of the system variables and the ones that are measured for the three considered motion control sub-systems.

\section{Mechanical System Model}

The mechanical system is modeled using the multibody library in MapleSim ${ }^{\mathrm{TM}}$. The model includes the main components shown in Fig. 1 as well as the hydraulic cylinders for the three crane jibs.

In terms of kinematic structure, the crane can be treated as a large mechanism consisting of both rigid and flexible links connected by revolute joints. The structural flexibility of a crane similar to the one considered here has been studied by Henriksen et al. (2011) and Bak et al. (2011). It was shown that the flexibility of certain structural members can have a significant influence on the overall dynamic behavior of the crane and therefore must be accounted for in a dynamic model.

Here the finite segment method is used to model the flexibility. The main advantage of this method is that it is based on rigid body modeling techniques, making it easy to implement. The method was originally developed by Huston (1981) and further studied in Huston (1991), Huston and Wang (1993), Connelly and Huston (1994a) and Connelly and Huston (1994b). Hansen et al. (2001) used the method for modeling of a mobile crane and achieved encouraging results in terms of conformity between measurements and simulations.
The masses, inertias and geometry of the mechanical components have been extracted from CAD models.

\subsection{Kinematic Structure}

The topology of the crane is globally an open kinematic chain, formed by the crane jibs, with locally closed chains formed by the hydraulic cylinders. With the main components and the barrels and pistons of the three hydraulic cylinders, the model includes a total of 12 bodies, see Fig 4.

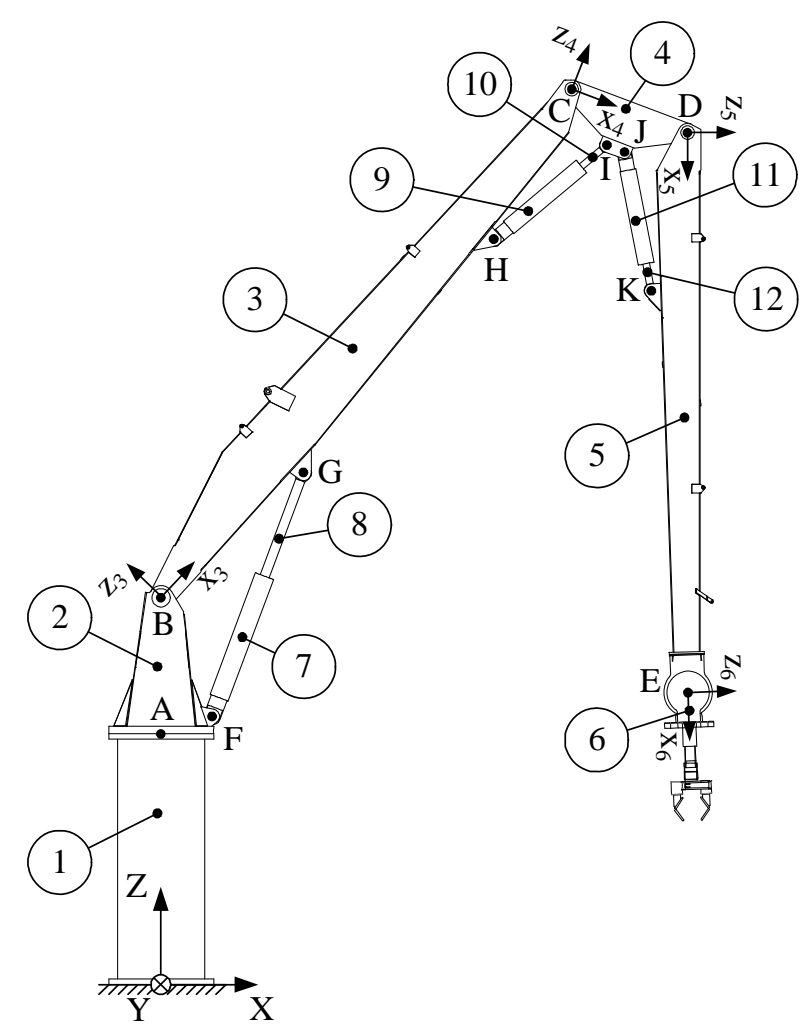

Figure 4: Topology of mechanical system model.

Since the slewing DOF of the crane is not considered, the crane can be modeled as a planar mechanism using revolute and translational joints. However, only spatial multi-body elements are available in MapleSim ${ }^{\mathrm{TM}}$, making the selection of kinematic constraints less straight forward. To identify a suitable kinematic structure the crane is initially treated as a rigid body system.

The bottom of the pedestal is fixed at the global origin and the king is fixed to the top of the pedestal in point A. Points B, C, D and E represent revolute joints (RJ). The connection points of cylinder barrels, points F, H and J, are modeled as spherical joints (SJ) while the connection points for pistons, points $\mathrm{G}, \mathrm{I}$ and 
$\mathrm{K}$, are modeled as universal joints (UJ). The translational DOFs between the cylinder barrels and pistons are modeled as prismatic joints (PJ). This leaves four remaining DOFs (Nikravesh, 1988):

$$
\begin{aligned}
n_{D O F}= & 6 \cdot n_{\text {bodies }}-6 \cdot n_{\text {fixtures }}-5 \cdot n_{P J} \\
& -5 \cdot n_{R J}-4 \cdot n_{U J}-3 \cdot n_{S J} \\
= & 72-12-15-20-12-9=4
\end{aligned}
$$

These are the ones of the three crane jib, which are actuated by hydraulic cylinders, and the rotational DOF of the gripping yoke (point E). The latter is actuated by a hydraulic motor, which is not considered here. The DOF is included to be able to orientate and fix the gripping in the wanted positions.

\subsection{Flexibility}

When applying the finite segment method to a planar mechanism, the flexible members are divided into a number of rigid segments which are connected by revolute joints and rotational springs (and dampers), see Fig. 5.
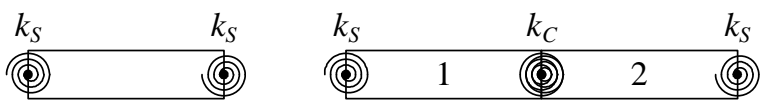

Figure 5: Concept of the finite segment method.

The left part of the figure illustrates a model of a single segment. The flexibility of the segment is represented by two rotational springs, both with the stiffness $k_{S}$. In the right part of the figure two segments are connected. The stiffness $k_{C}$ of the connection between the segments corresponds to a series connection of the springs for the two adjoining segments:

$$
k_{C}=\frac{1}{\frac{1}{k_{S, 1}}+\frac{1}{k_{S, 2}}}
$$

$k_{S, 1}$ is the segment stiffness of the segment 1 and $k_{S, 2}$ is the segment stiffness of the segment 2 .

For bending the segment stiffness is:

$$
k_{S}=\frac{2 \cdot E \cdot I}{L}
$$

$E$ is the Young's modulus, $I$ is the area moment of inertia and $L$ is the length of the segment.

The pedestal, the inner jib and the outer jib are assumed to dominate the overall structural flexibility of the crane since the remaining components are far more compact. Fig. 6 shows the segmentation of the three flexible members.

The pedestal, inner jib and outer jib (body numbers 1 ,

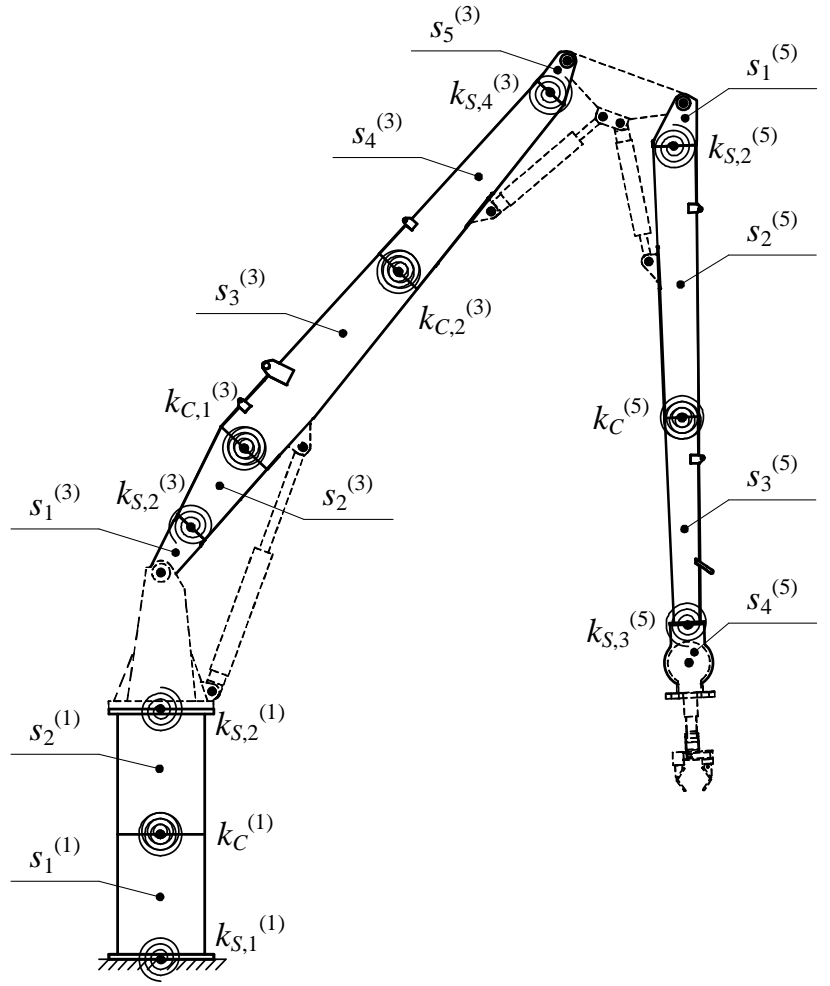

Figure 6: Segmentations of flexible members.

3 and 5) are divided into two, five and four segments, respectively. The number of segments is certainly debatable, but is kept low for computational reasons.

As seen from Fig. 6, the inner and outer jibs are beamlike structures with varying cross sections. With the chosen segmentations, the cross section areas of the individual segments vary linearly and continuously and therefore average values of the segments area moments of inertia are used to determine the segment stiffnesses, $k_{S}$. The area moments of intertia at the cross sections between the segments are extracted from CAD models and used to determine the average area moments of inertia. Henriksen et al. (2011) used this approach for a model of a single crane jib and compared both static deflections and natural frequencies of a finite segment model with results of a finite element analysis and achieved a remarkable conformity even without calibrating the model.

The segment and connection stiffnesses, determined using (2) and (3), are given in Table 2. The first and last segments of the inner and outer jibs are assumed rigid and therefore the stiffness, $k_{S}$, of the neighboring segment is used as the connection stiffness, $k_{C}$.

The stiffnesses in the applied lumped parameter model are indeed uncertain (soft) parameters and (2) and (3) merely represent a means to estimate those parameters. As argued by Shabana (1997), they can also be 
Table 2: Segment and connection stiffnesses.

\begin{tabular}{llll}
\hline Segment & $k_{S}[\mathrm{Nm} / \mathrm{rad}]$ & $k_{C}[\mathrm{Nm} / \mathrm{rad}]$ \\
\hline$s_{1}^{(1)}$ & $5.75 \cdot 10^{9}$ & $2.88 \cdot 10^{9}$ & \\
$s_{2}^{(1)}$ & $5.75 \cdot 10^{9}$ & & \\
\hline$s_{1}^{(3)}$ & rigid & $1.96 \cdot 10^{9}$ & \\
$s_{2}^{(3)}$ & $1.96 \cdot 10^{9}$ & & $6.03 \cdot 10^{8}$ \\
$s_{3}^{(3)}$ & $8.73 \cdot 10^{8}$ & $2.77 \cdot 10^{8}$ & \\
$s_{4}^{(3)}$ & $4.05 \cdot 10^{8}$ & & $4.05 \cdot 10^{8}$ \\
$s_{5}^{(3)}$ & rigid & & \\
\hline$s_{1}^{(5)}$ & rigid & $2.08 \cdot 10^{8}$ & \\
$s_{2}^{(5)}$ & $2.08 \cdot 10^{8}$ & & $8.33 \cdot 10^{7}$ \\
$s_{3}^{(5)}$ & $1.39 \cdot 10^{8}$ & $1.39 \cdot 10^{8}$ & \\
$s_{4}^{(5)}$ & rigid & & \\
\hline
\end{tabular}

determined by finite element analysis and parameter identification. This is, however, not the scope of this paper and instead the stiffnesses are tuned during the calibration (section 5) of the entire crane model.

\subsection{Damping}

The dynamic characteristics of the mechanical model depend on the damping applied to it and therefore the damping must be carefully considered. However, damping parameters are even more uncertain than those related to the flexibility. The only way to properly determine the damping parameters may be to carry out a thorough experimental study of the considered structure. As in this case, time, resources and practical circumstances seldom allow for that kind of investigations.

Therefore, whether working with design models or models for analysis and with limited possibilities for experimental investigations, there is a need for methods to determine the damping parameters with a reasonable accuracy. Mostofi (1999) presents a simple approach that can be used for lumped parameter modeling techniques such as the finite segment method. For stiffness damping (damping elements in parallel with the flexible elements), the damping of a structural member is:

$$
\mathbf{c}=\beta_{k} \cdot \mathbf{k}
$$

$\mathbf{k}$ contains the spring coefficients determined in section 3.2 and $\beta_{k}$ is a stiffness multiplier determined by:

$$
\beta_{k}=\frac{2 \cdot \zeta_{S}}{\omega_{S}}
$$

$\zeta_{S}$ is the damping ratio of the structure and $\omega_{S}$ is the natural frequency of the structure for the considered mode of motion. Representative damping ratios for different structures are given in Adams and Askenazi (1999). For metal structures with joints, e.g., weldings and bolted connections, the damping ratio is $\zeta=0.03-0.07$. Naturally, this is subject to uncertainty but, nevertheless, better than a simple guess.

To determine the damping coefficients for the models of the three flexible members, simulations without damping have been carried out to find the natural frequency of each member. In the simulations each member is fixed in one end (cantilevered) and an impulse is applied to excite oscillations from which the natural frequency can be observed. The natural frequencies and corresponding range of stiffness multipliers for the three flexible members are given in table 3 .

Table 3: Natural frequencies and stiffness multipliers.

\begin{tabular}{lll}
\hline Member & $\omega_{S}[\mathrm{rad} / \mathrm{s}]$ & $\beta_{k}[\mathrm{~s}]$ \\
\hline Pedestal (with king) & 94 & $0.0006-0.0015$ \\
Inner jib & 60 & $0.001-0.002$ \\
outer jib & 35 & $0.002-0.004$ \\
\hline
\end{tabular}

The stiffness multipliers are tuned together with spring coefficients during the model calibration in section 5 .

\section{Motion Control System Model}

Whereas the model of the mechanical system, in general, is based on physical (white-box) modeling, the motion control system model is mostly based on semiphysical (grey-box) modeling. The main reason for this is that manufacturers of hydraulic components do not provide enough and sufficiently detailed information to establish physical models. In addition, physical models will quickly become too complex and computational demanding for system simulation. Therefore certain model structures have to be assumed which allow for simplifications without ignoring or underestimating important physical phenomena.

The motion control system is modeled using both predefined components from the hydraulic and the signal block libraries in MapleSim ${ }^{\mathrm{TM}}$ and custom made components developed via Maple ${ }^{\mathrm{TM}}$. This combination facilitates both efficient model development and modeling at a detail level that is not supported by library components.

Joystick commands and control signals are represented by normalized signal, which can vary continuously between -1 and 1 , and component dynamics is modeled using transfer functions.

Hydraulic valves are generally modeled as variable orifices with linear opening characteristics:

$$
Q=\xi \cdot C_{V} \cdot \sqrt{\Delta p}
$$


Here $\xi$ is the relative opening of the valve, i.e., a dimensionless number between 0 and 1 . It can be a function of system pressures or controlled with an input signal depending on the considered type of valve. $Q$ is the volume flow through the valve and $\Delta p$ is the pressure drop across it.

The flow coefficient in (6) can be expressed as:

$$
C_{V}=C_{d} \cdot A_{d} \cdot \sqrt{\frac{2}{\rho_{\text {oil }}}}
$$

The discharge coefficient, $C_{d}$, and the discharge area, $A_{d}$, are usually not specified for a valve. Instead $C_{V}$ can be obtained from characteristic flow curves given in the datasheet of the valve. From this, a nominal flow, $Q_{\text {nom }}$, corresponding to a nominal pressure drop, $\Delta p_{\text {nom }}$, can be identified and used to derive the flow coefficient:

$$
C_{V}=\frac{Q_{n o m}}{\sqrt{\Delta p_{n o m}}}
$$

This corresponds to the fully opened state of the valve, so with (6) it is assumed that the discharge coefficient, $C_{d}$, is constant and only the discharge area, $A_{d}$, varies with the relative opening of the valve.

This modeling approach works well for DCVs with closed loop spool position control where dither is used to eliminate static friction and certain design details are used to reduce the disturbances from flow forces. In some cases the approach may also work for pressure control valves like CBVs. Most often, though, attempting to establish physical or semi-physical models of such valves will encounter a number of challenges, e.g., related to friction and resulting hysteresis, nonlinear discharge area characteristics, varying discharge coefficients and varying flow forces. Therefore, a better way to model those types of valves may be to use a non-physical (black-box) approach as described in section 4.2.

The following sub-sections describe models of the DCVs, the CBVs and the hydraulic cylinders.

\subsection{Directional Control Valves}

The main concern, when modeling a DCV, is the steady-state flow characteristics and the dynamics (bandwidth) of the valve. For system simulations, the design details of the valve are usually not important and therefore servo valves and pressure compensated DCVs can often be represented by the same model. Breaking down the model into several elements offers flexibility and facilitates changes in the model like including or excluding a pressure compensator. The general DCV model includes a representation of the valve actuation (pilot stage) and four elements for the main spool. For pressure compensated valves, the model also includes the LS circuit and the pressure compensator. The model structure for the pressure compensated DCV is shown in Fig. 7. The blue lines are signal lines transferring only a single state variable. The red lines transfer the two hydraulic state variable, pressure and flow, between the hydraulic components. These are custom made components developed in Maple ${ }^{\mathrm{TM}}$.

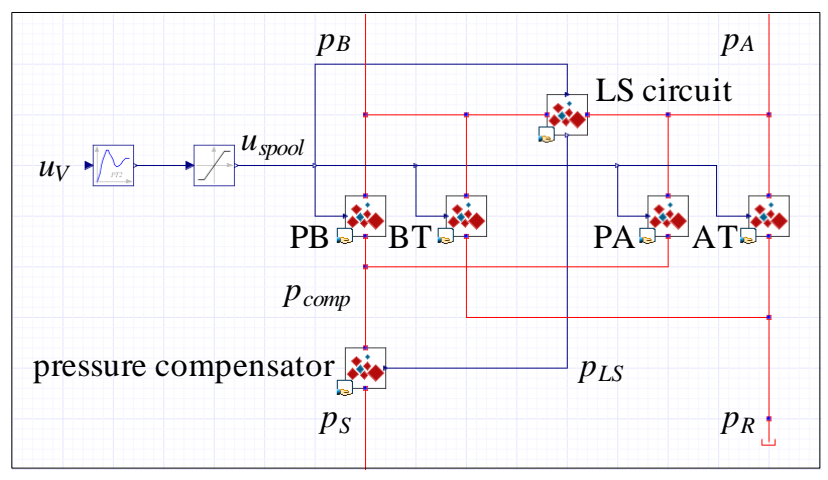

Figure 7: Structure of DCV model.

The valve is actuated with the control signal $u_{V}$, which is passed through a second order system representing the dynamics of the valve:

$$
\frac{u_{\text {spool }}}{u_{V}}=\frac{1}{\frac{s^{2}}{\omega_{V}^{2}}+2 \cdot \zeta_{V} \cdot \frac{s}{\omega_{V}}+1}
$$

The output, $u_{\text {spool }}$, is a normalized signal representing the spool position, which can vary continuously between -1 and 1 with 0 being the center position of the spool. The natural frequency, $\omega_{V}$, represents the bandwidth of the valve and $\zeta_{V}$ is the damping ratio.

For servo valves the bandwidth can usually be identified from the valves datasheet. Here the valve dynamics is usually visualized with Bode plots for several input amplitudes showing that the dynamics is non-linear. For system simulations, though, a linear model like the second order system will most often be sufficient to capture the dominant dynamics.

For pressure compensated DCVs there is usually no information available about the bandwidth and the only way to identify it may be to carry out a frequency response test of the considered valve. An approach for such a test is described in Bak and Hansen (2012) along with some test results for a Danfoss PVG32, which is identical to the DCVs used for the intermediate and outer jibs. The identified bandwidth, $\omega_{V}=30 \mathrm{rad} / \mathrm{s}$, and damping ratio, $\zeta_{V}=0.8$, are therefore also used here. This represents the overall dynamics between the control signal and the controlled flow, i.e., the dynamics of the pilot stage, the main spool, the LS circuit and the pressure compensator. 
The four main spool edges are modeled as variable orifices, according to (6), for which the relative opening, $\xi_{\text {edge }}$, of each spool edge is a function of the normalized spool position signal as shown in Fig. 8.

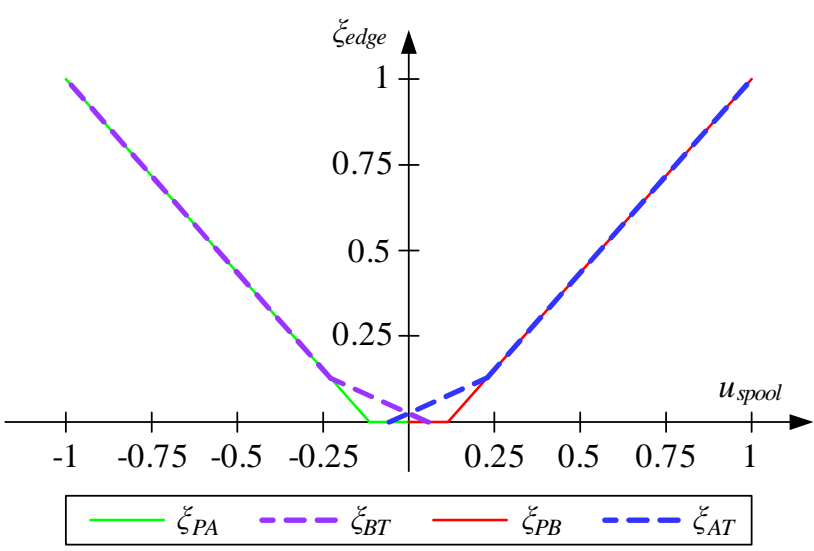

Figure 8: Opening functions for spool edges.

The spool edge openings are piecewise linear functions that include the overlaps of the metering edges, PB and $\mathrm{PA}$, and the underlaps of the return edges, AT and BT. A more detailed description of the opening functions is given in Bak and Hansen (2012).

When modeling pressure compensated DCVs, a problem often encountered is that very little or no information about the pressure compensator is available from the valves datasheet. This makes it difficult to estimate when the pressure saturation will occur and to establish a model of the compensator. However, if the nominal pressure drop across the main spool metering edge (setting of compensator spring), $p_{0}$, is known the compensated pressure, $p_{\text {comp }}$, can be described as:

$$
p_{c o m p}=\left\{\begin{array}{lll}
p_{L S}+p_{0} & \text { for } \quad p_{0} \leq p_{S}-p_{L S} \\
p_{S}-p_{L S} & \text { for } \quad 0<p_{S}-p_{L S}<p_{0} \\
p_{L S} & \text { for } \quad p_{S}-p_{L S} \leq 0
\end{array}\right.
$$

The first case describes the normal operating condition of the compensator, maintaining the nominal pressure drop across the main spool metering edge. The second case describes the condition where the load pressure is too close to the supply pressure to maintain the nominal pressure drop. The third case describes the build-in check valve function of the compensator, which prevents negative flow if the load pressure exceeds the supply pressure.

The LS circuit directing the load pressure to the pressure compensator is modeled as a piecewise function:

$$
p_{L S}= \begin{cases}p_{A} & \text { for } u_{\text {spool }}<0 \\ p_{B} & \text { for } 0<u_{\text {spool }} \\ 0 & \text { otherwise }\end{cases}
$$

The model parameters for the servo valve (inner jib) and the pressure compensated DCVs (intermediate and outer jibs) are given in Table 4.

Table 4: DCV model parameters.

\begin{tabular}{lll}
\hline & Inner jib & Int. \& outer jibs \\
\hline$\omega_{V}$ & $250 \mathrm{rad} / \mathrm{s}(40 \mathrm{~Hz})$ & $30 \mathrm{rad} / \mathrm{s}(5 \mathrm{~Hz})$ \\
$\zeta_{V}$ & 0.8 & 0.8 \\
Overlap & $5 \%$ & $10 \%$ \\
Underlap & 0 & $5 \%$ \\
$C_{V}$ & $111.8 \frac{l}{\text { min.bar }^{0.5}}$ & $37.8 \frac{l}{\text { min.bar }}$ \\
$p_{0}$ & $\mathrm{NA}$ & $7 \mathrm{bar}$ \\
\hline
\end{tabular}

\subsection{Counterbalance Valves}

The model of the CBV consists of two components; a check valve and a pilot assisted relief valve (the actual CBV).

The check valve is modeled according to (6) with the relative opening given by:

$$
\xi_{C V}=\frac{p_{1}-p_{2}-p_{c r, C V}}{k_{s, C V}}
$$

This is only the basic part of a piecewise function that limits the relative opening to the interval $\xi_{C V}=[0,1]$. $p_{1}$ is the pressure at the port connected to the DCV and $p_{2}$ is the pressure at the port connected to the cylinder. The cracking pressure, $p_{c r, C V}$, is usually specified for a CBV and the normalized spring stiffness can be set to a low value of, e.g., $k_{s, C V}=1$ bar.

In practice the check valve is either opened or closed. The spring stiffness is only used to handle the transition between the two states, which otherwise may cause computational difficulties.

Describing the relative opening by means of a pressure equilibrium like (12) can in some cases also be used for the CBV itself. However, for the previously mentioned reasons, it is often only valid for the condition where the CBV is just about to open (crack). For an externally vented CBV, like the one in Fig. 2, the cracking condition is described by:

$$
p_{2}+\psi \cdot p_{x}=p_{c r, C B V}
$$

Once again, $p_{2}$ is the pressure at the port connected to the cylinder. $p_{x}$ is the pilot pressure and $\psi$ is the pilot area ratio.

For a non-vented CBV, like the one in Fig. 3 (ignoring the two internal orifices), the cracking condition is:

$$
p_{2}+\psi \cdot p_{x}=p_{c r, C B V}+(1+\psi) \cdot p_{1}
$$


As for the check valve, $p_{1}$ is the pressure at the port connected to the DCV.

Instead of using (6) and a pressure equilibrium to model the CBV it is proposed to use a black-box approach based on the cracking condition in (13).

For the black-box model, two new variables are introduced; $\mu_{x}$ and $\mu_{L}$. The first one is the ratio between the pilot pressure, $p_{x}$, and the load pressure, $p_{L}$ :

$$
\mu_{x}=\frac{p_{x}}{p_{L}}
$$

The second variable is dependent on the type of CBV. For an externally vented CBV, it is the ratio between the load pressure and the cracking pressure:

$$
\mu_{L}=\frac{p_{L}}{p_{c r, C B V}}
$$

For a non-vented CBV, it is the ratio between the pressure drop across the valve and the cracking pressure:

$$
\mu_{L}=\frac{\Delta p}{p_{c r, C B V}}
$$

By replacing $p_{2}$ with $p_{L}$ in (13) it can be combined with (15) and (16) to arrive at the following expression:

$$
\mu_{L, c r}=\frac{1}{1+\psi \cdot \mu_{x}}
$$

This expression, just as (13) and (14), describes the cracking condition of the CBV where there is still no flow the through the valve. It is illustrated by the solid line in Fig. 9, here for a $\mathrm{CBV}$ with a pilot area ratio of $\psi=3$. The dashed lines above illustrate conditions with different levels of flow through the valve.

In the model the actual $\mu_{x}$ and $\mu_{L}$ for a given time step is computed by (15) and either (16) or (17) depending on the type of CBV. The distance, $s$, from the cracking line (randomly shown in Fig. 9) is then computed:

$$
s=\mu_{L}-\mu_{L, c r}
$$

If the distance is negative, there is no flow through the valve. Otherwise, the flow is computed as:

$$
Q_{C B V}=A_{C B V} \cdot s^{n_{C B V}}
$$

Here $A_{C B V}$ is given by:

$$
A_{C B V}=A_{0}+A_{1} \cdot \mu_{x}
$$

Similarly, $n_{C B V}$ is given by:

$$
n_{C B V}=n_{0}+n_{1} \cdot \mu_{x}
$$

The four parameters, $A_{0}, A_{1}, n_{0}$ and $n_{1}$ have to be experimentally determined, ideally by a thorough

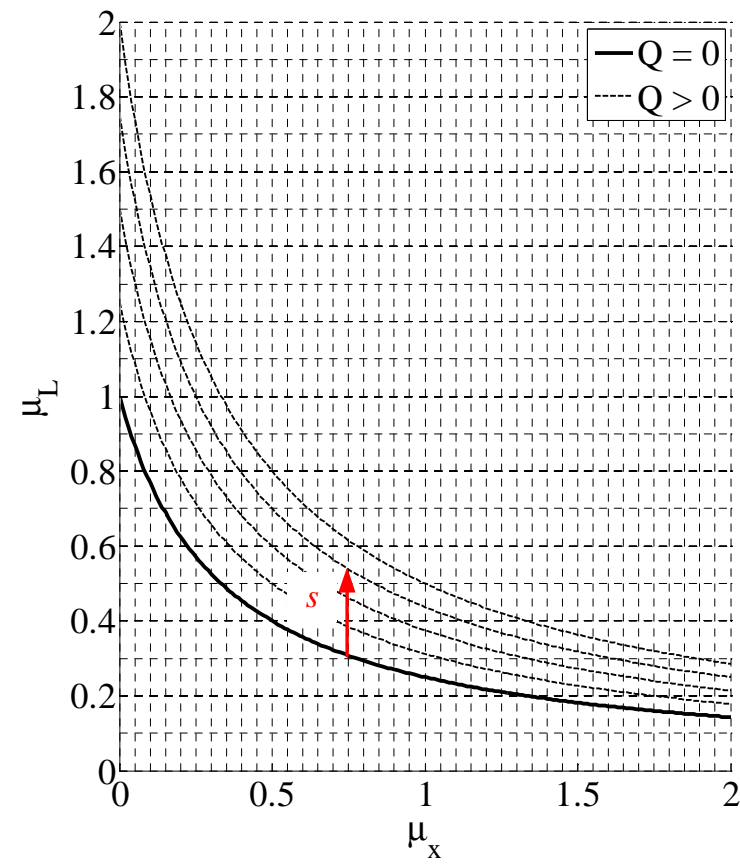

Figure 9: Example of the relation between $\mu_{L}$ and $\mu_{x}$ for the CBV model.

mapping of the flow through the CBV for different pressure combinations at the individual ports. Alternatively, as described in section (5.2), they can be determined with parameter identification techniques and suitable measurements from the system where the $\mathrm{CBV}$ is installed.

The remaining (known) model parameters for the CBVs and the check valves for the inner jib and the intermediate and outer jibs are given in Table 5 .

Table 5: CBV model parameters.

\begin{tabular}{lll}
\hline & Inner jib & Int. \& outer jibs \\
\hline$C_{V}$ & $89.4 \frac{l}{\min \cdot b a r^{0.5}}$ & $63.2 \frac{l}{\min \cdot b a r^{0.5}}$ \\
$p_{c r, C V}$ & $1 \mathrm{bar}$ & $1 \mathrm{bar}$ \\
$k_{s, C V}$ & $1 \mathrm{bar}$ & $1 \mathrm{bar}$ \\
$p_{c r, C B V}$ & $250 \mathrm{bar}$ & $250 \mathrm{bar}$ \\
$\psi$ & 3 & 6 \\
\hline
\end{tabular}

\subsection{Hydraulic Cylinders}

The model of the hydraulic cylinder includes the capacitance of the chambers as well as the friction between 
the piston and the barrel. The cylinder force is:

$$
F_{c y l}= \begin{cases}F_{p}-F_{f r} & \text { for } \quad v_{c y l}<v_{0} \\ F_{p}-F_{f r} \cdot \frac{v_{c y l}}{v_{0}} & \text { for } \quad-v_{0} \leq v_{c y l} \leq v_{0} \\ F_{p}+F_{f r} & \text { for } \quad v_{c y l}<-v_{0}\end{cases}
$$

The pressure force is $F_{p}=p_{1} \cdot A_{p}-p_{2} \cdot \phi \cdot A_{p}$. Here $A_{p}$ is the piston area and $\phi=\left(D_{p}^{2}-D_{r}^{2}\right) / D_{p}^{2}$, where $D_{p}$ is the piston diameter and $D_{r}$ is the rod diameter. $p_{1}$ and $p_{2}$ are the pressures in the corresponding cylinder chambers. $v_{c y l}$ is the cylinder piston velocity and $v_{0}$ is a transition velocity used to handle the change in friction force around zero velocity.

The capacitance of the two chamber volumes are accounted for by:

$$
\begin{aligned}
& \dot{p}_{1}=\frac{\beta_{o i l}}{V_{1}} \cdot\left(Q_{1}-v_{c y l} \cdot A_{p}\right) \\
& \dot{p}_{2}=\frac{\beta_{o i l}}{V_{2}} \cdot\left(v_{c y l} \cdot A_{a}-Q_{2}\right)
\end{aligned}
$$

$\beta_{\text {oil }}$ is the effective stiffness (bulk modulus) of the hydraulic fluid. $Q_{1}$ and $Q_{2}$ are volume flows in the two cylinder chambers. The chamber volumes, $V_{1}$ and $V_{2}$, are functions of the cylinder length.

The friction in the cylinder is quite complex, especially around zero velocity. As described in Ottestad et al. (2012) it consists of both static and Coulomb friction as well as velocity dependent and pressure dependent friction, which may be described with a model of five parameters. Even though the model is not very complex, the number of parameters represents a problem because they cannot be determined without an extensive experimental study of the considered cylinder.

Consequently, an even simpler model must be used and therefore the friction force in (23) consists only of static friction and pressure dependent friction:

$$
F_{f r}=F_{S}+C_{p} \cdot\left|F_{p}\right|
$$

The static friction can be set to $F_{S}=A_{p} \cdot 1 \cdot 10^{5} \mathrm{~m}^{2} \cdot \mathrm{Pa}$, i.e., a pressure of 1 bar on the piston-side is required to overcome the static friction. The pressure dependent friction may constitute $2 \ldots 3 \%$ of the hydraulic force, e.g., $C_{p}=0.02$. The friction parameters are identified in section 5. The remaining (known) parameters for the cylinders are given in Table 6 .

\section{Parameter Identification}

For verification of the model, experiments have been carried out where inputs, outputs and certain state variables (given in section 2) of the real system have been measured and recorded. The crane used for the
Table 6: Cylinder model parameters.

\begin{tabular}{lll}
\hline & Inner jib & Int. \& outer jibs \\
\hline$D_{p}$ & $0.3 \mathrm{~m}$ & $0.25 \mathrm{~m}$ \\
$D_{r}$ & $0.18 \mathrm{~m}$ & $0.125 \mathrm{~m}$ \\
$l_{0}$ & $3.145 \mathrm{~m}$ & $2.315 \mathrm{~m}$ \\
stroke & $1.755 \mathrm{~m}$ & $1.33 \mathrm{~m}$ \\
mass & $1500 \mathrm{~kg}$ & $750 \mathrm{~kg}$ \\
\hline
\end{tabular}

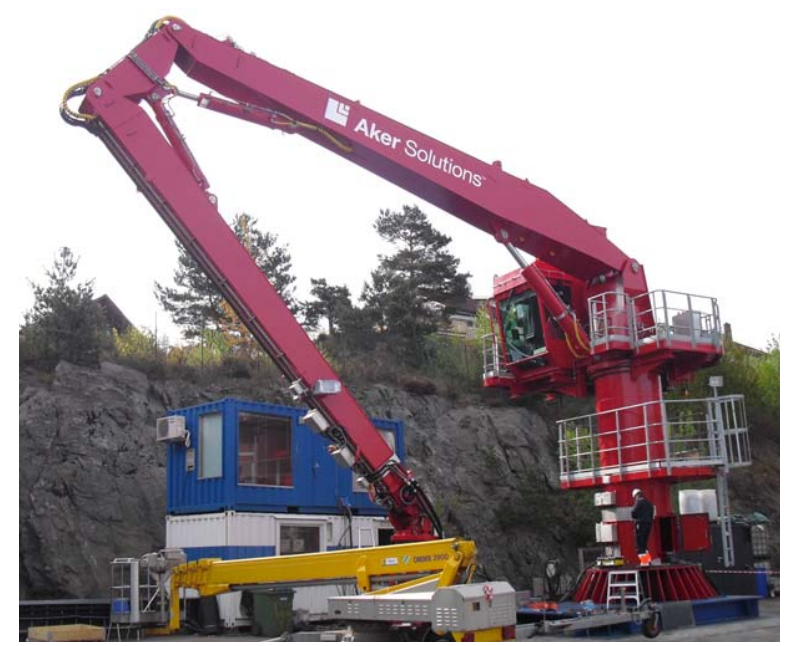

Figure 10: Crane used for experiments.

experimental work is shown in Fig. 10.

In order to calibrate and verify the model, the inputs from the experiments are fed to the model and the uncertain parameters are systematically tuned (identified) until both simulated outputs and state variables correspond to those obtained in the experiments. The model can then be considered as verified as illustrated in Fig. 11.

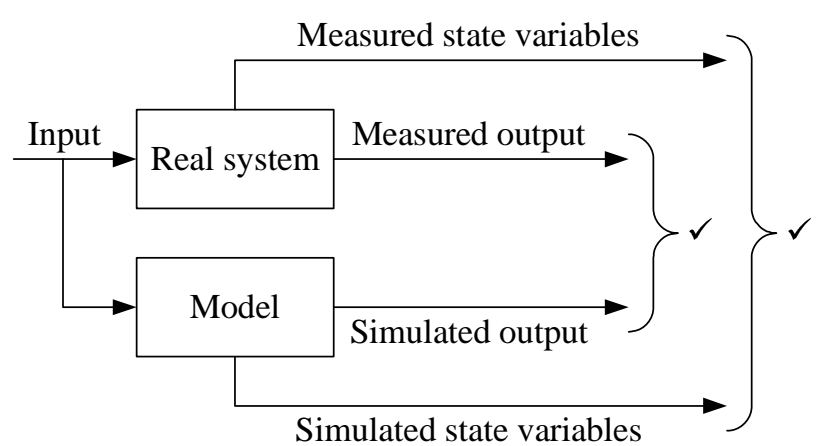

Figure 11: Principle of model verification.

To simplify the experiments and the following parameter identification, the individual DOFs are considered 

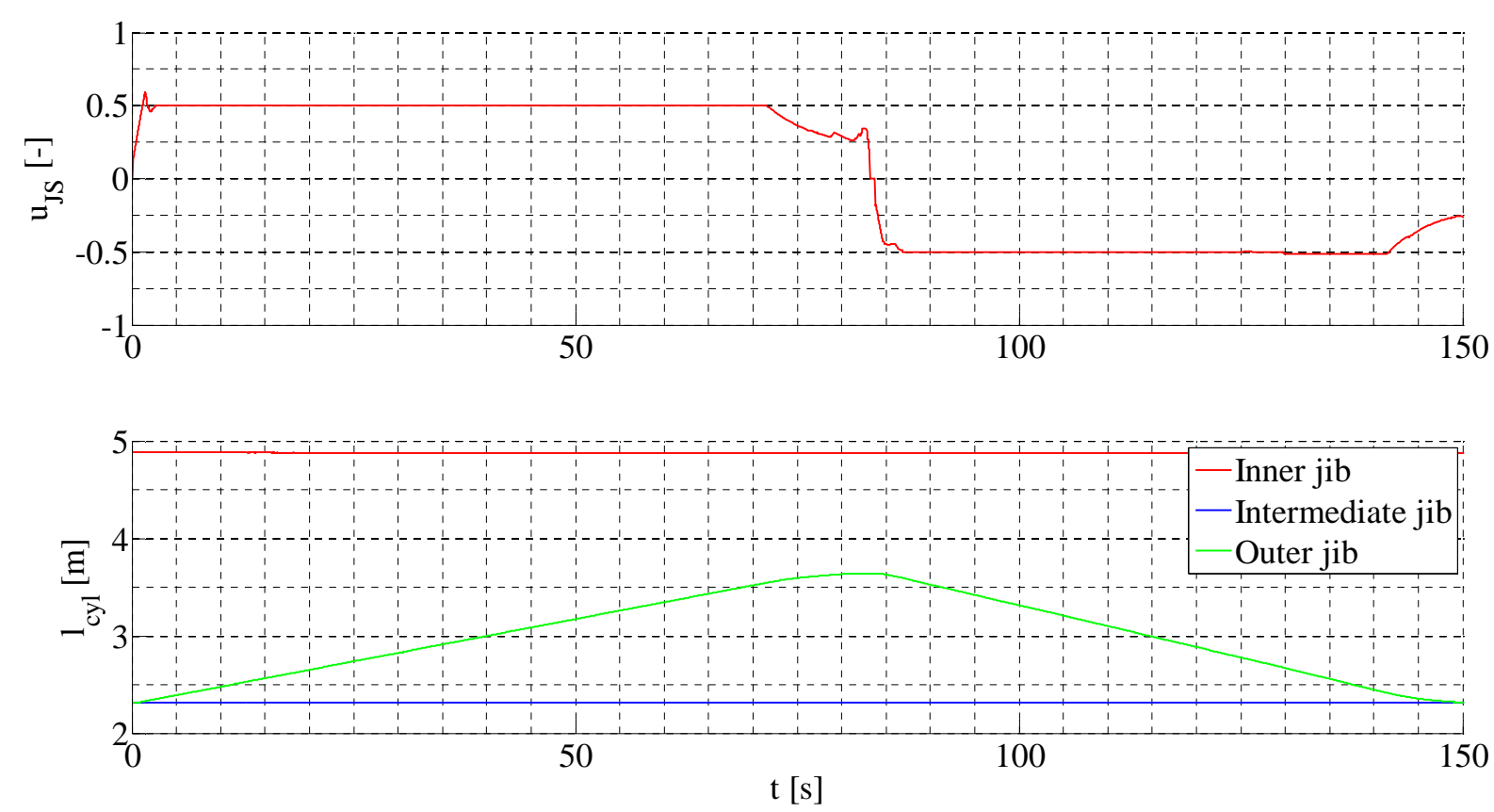

Figure 12: Experimental input and output for motion control sub-system for outer jib.

separately and one at the time. Since the procedure for identifying the parameters and verifying the model is the same for all three DOFs, only the DOF of the outer jib is considered in the following. During the experimental procedure the crane is operated in open loop control mode, i.e., joystick signals are passed directly to the control valve of the considered DOF. The joystick signal and the resulting cylinder motion for the outer jib DOF are shown in Fig. 12.

The calibration of the model is carried out in three steps; first the steady-state cylinder forces are considered, next the steady-state pressures and finally the system dynamics are considered.

Both steady-state and dynamic simulation is used for the model calibration. Steady-state simulation is carried out in MATLAB $^{\circledR}$, and dynamic simulation is carried out in Simulink ${ }^{\circledR}$ using an S-function (compiled C-code) generated from the MapleSim ${ }^{\mathrm{TM}}$ model. The main advantage of this approach is a significant increase in simulation speed. Furthermore, simulated values are quickly compared with measured values by importing the latter from the MATLAB ${ }^{\circledR}$ workspace and plotting them together with the simulated values. The Simulink ${ }^{\circledR}$ model is shown in Fig. 13.

The pressures in the circuit of the considered DOF is monitored and the measured pressures are compared with the simulated pressures. Simulated and measured lengths of all three cylinders are monitored for verifi-

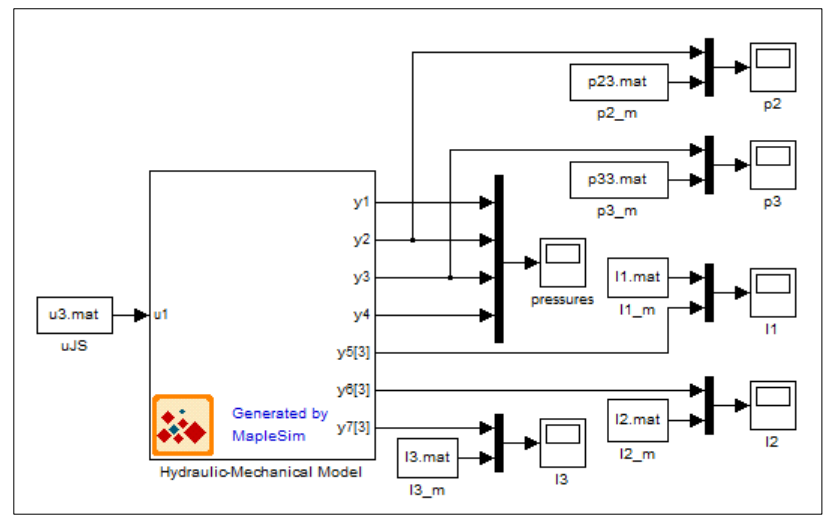

Figure 13: Simulink model of the crane.

cation, i.e., to calibrate the motion of the considered cylinder and to ensure that the two remaining cylinders are not moving during simulation.

\subsection{Steady-State Forces}

A prerequisite for verification of the motion control system is to verify the mechanical model and to identify its uncertain parameters. For this purpose a rigid body steady-state model of the mechanical system, as shown in Fig. 4, is used to simulate the steady-state pressure forces in the cylinders, in order to compare these with 
the measured pressure forces. The simulation is carried out by using the measured cylinder motion given in Fig. 12 as input and computing the resulting steadystate force on the cylinder.

A parameter identification is carried out to identify the friction parameters for the cylinder model and to tune the mechanical model parameters, i.e., the masses and positions of the centers of gravity (COGs) originally found via CAD models. Since a CAD model seldom takes into account all the components in a mechanical assembly it is reasonable to allow for a tuning of the mechanical properties within a given set of constraints.

The parameter identification is an optimization routine, based on the fmincon function in MATLAB ${ }^{\circledR}$, which minimizes the squared deviation between the measured pressure force, $\widetilde{F}_{p}$, and the simulated pressure force, $F_{p}$ :

$$
\text { minimize } f=\left(\widetilde{F}_{p}-F_{p}\right)^{2}
$$

The simulated pressure force is computed as:

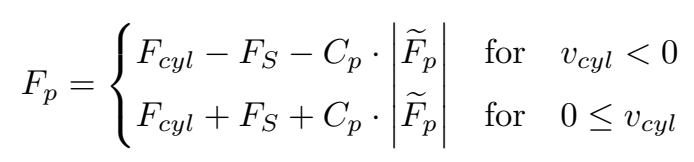

$F_{c y l}$ is the simulated steady-state force on the cylinder and $v_{c y l}$ is the measured cylinder piston velocity.

The measured pressure force and simulated forces after the parameter identification are shown in Fig. 14. The parameter values before and after the identification are given in Table 7. As the pedestal and the king (bodies 1 and 2) are stationary, they are not included in the parameter identification. The positions of the COGs are according to the local coordinate systems in Fig. 4 The steady-state levels of the measured and simulated pressure forces are nearly equal and the mechanical model along with the identified parameters are therefore considered reliable.

\subsection{Steady-State Pressures}

The verification of the steady-state behavior of the control system model mainly depends on the calibration of the CBV model, i.e., identification of the four model parameters in (21) and (22). For that the flows, $Q_{1}$ and $Q_{2}$, through $\mathrm{CBV}_{1}$ and $\mathrm{CBV}_{2}$ are computed from the measured cylinder motion shown in Fig. 12 and used in a steady-state model of the outer jib circuit, together with the two measured pressures, $p_{2}$ and $p_{3}$, in order to estimate the two remaining pressures, $p_{1}$ and $p_{4}$. The measured and estimated state variables are then used together with the CBV model to identify $A_{0}, A_{1}, n_{0}$ and $n_{1}$.

The two CBVs in the outer jib circuit are identical with
Table 7: Mechanical model parameters before and after parameter identification. Positions of COGs are according to Fig. 4.

\begin{tabular}{lll}
\hline Parameter & Before & After \\
\hline$m_{3}$ & $5500 \mathrm{~kg}$ & $5600 \mathrm{~kg}$ \\
$m_{4}$ & $1100 \mathrm{~kg}$ & $1150 \mathrm{~kg}$ \\
$m_{5}$ & $2950 \mathrm{~kg}$ & $3000 \mathrm{~kg}$ \\
$m_{6}$ & $2700 \mathrm{~kg}$ & $2710 \mathrm{~kg}$ \\
\hline$x_{3}$ & $5.3 \mathrm{~m}$ & $5.4 \mathrm{~m}$ \\
$z_{3}$ & $0.4 \mathrm{~m}$ & $0.35 \mathrm{~m}$ \\
$x_{4}$ & $1.15 \mathrm{~m}$ & $1.1 \mathrm{~m}$ \\
$z_{4}$ & $-0.2 \mathrm{~m}$ & $-0.15 \mathrm{~m}$ \\
$x_{5}$ & $4.9 \mathrm{~m}$ & $4.8 \mathrm{~m}$ \\
$z_{5}$ & $-0.26 \mathrm{~m}$ & $-0.21 \mathrm{~m}$ \\
$x_{6}$ & $1 \mathrm{~m}$ & $1.57 \mathrm{~m}$ \\
$z_{0}$ & 0 & 0 \\
\hline$F_{S}$ (outer jib) & - & $6.4 \mathrm{kN}$ \\
$C_{p}($ outer jib) & - & 0.029 \\
\hline
\end{tabular}

a geometric pilot area ratio of $\psi=6$. During operation a small amount of oil flows through the internal orifices of the active CBV, which causes a reduction of the actual pilot pressure, $p_{x, 1}$ or $p_{x, 2}$, compared to the external pressure, $p_{1}$ or $p_{4}$, at the pilot port. For practical reasons, the external pressure at the pilot port is often considered as the pilot pressure and therefore the orifices are said to lower the effective pilot area ratio. With the given sizes of the two orifices the effective pilot area ratio is theoretically $\psi=1.95$, which is represented by the dashed black line in Fig. 15. However, this is only valid when the flow through the orifices is turbulent and the back pressure is zero.

In practice, there is always a certain level of back pressure and the flow through the orifices may not follow the fully turbulent orifice equation at all times. Therefore the original cracking line for $\psi=1.95$ is corrected according to:

$$
\mu_{L, c r}=\frac{1}{1+C_{1} \cdot \mu_{x}+C_{2} \cdot \mu_{x}^{2}}
$$

The corrected cracking line, the solid black line in Fig. 15 , is adjusted by tuning $C_{1}$ and $C_{2}$ until it coincides with the actual $\mu_{L}$ values obtained with the measured and estimated state variables.

During extension of the cylinder $\mathrm{CBV}_{2}$ is active and $\mu_{L}$ is obtained by:

$$
\mu_{L, 2}=\frac{p_{3}-p_{4}}{p_{c r, C B V}}
$$

$\mu_{x}$ is obtained by:

$$
\mu_{x, 2}=\frac{p_{1}}{p_{3}}
$$




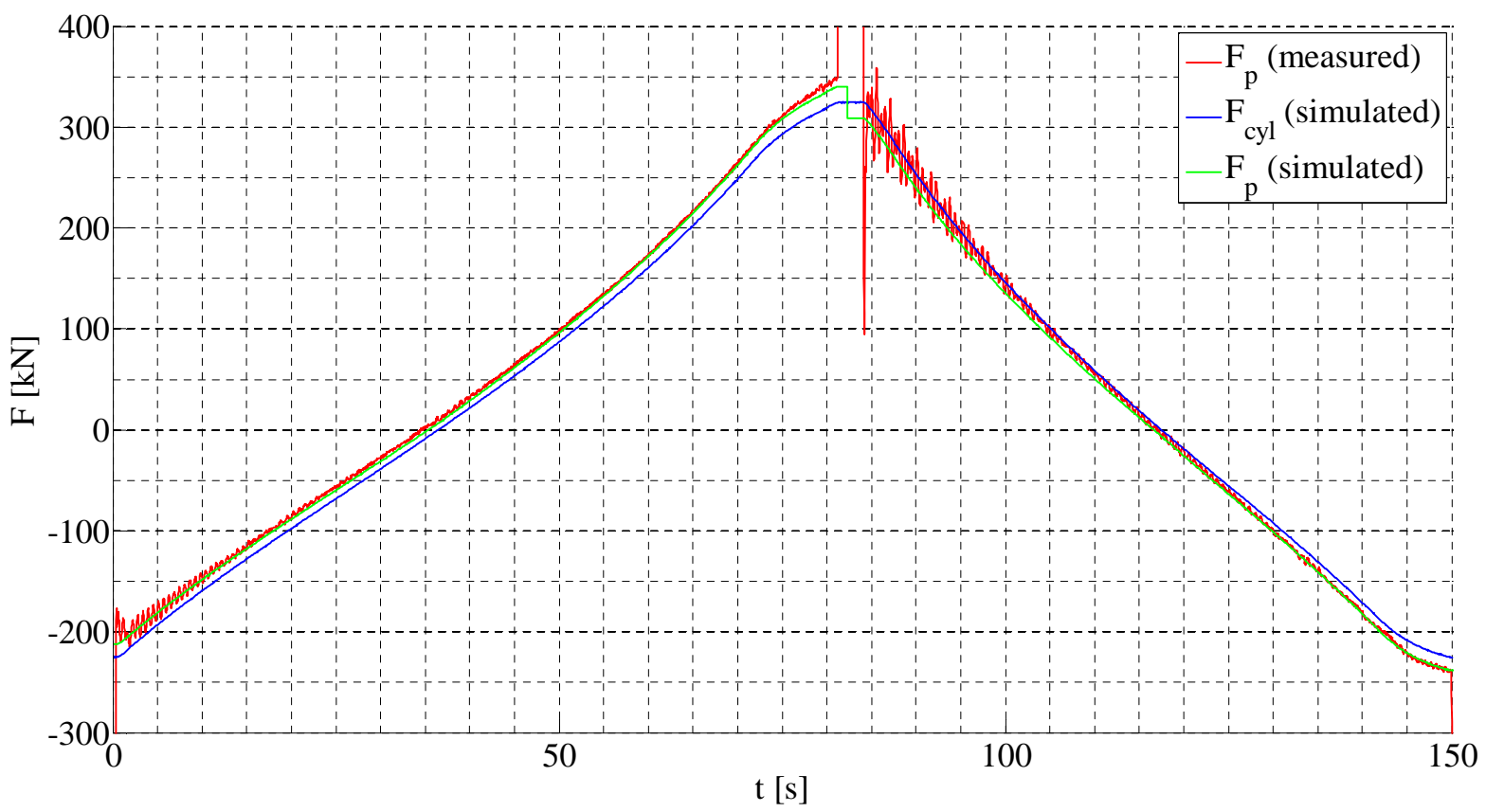

Figure 14: Measured and simulated forces for verification of steady-state characteristics.

During retracting of the cylinder $\mathrm{CBV}_{1}$ is the active one and $\mu_{L}$ is obtained by:

$$
\mu_{L, 2}=\frac{p_{2}-p_{1}}{p_{c r, C B V}}
$$

$\mu_{x}$ is obtained by:

$$
\mu_{x, 2}=\frac{p_{4}}{p_{2}}
$$

With the computed $\mu_{x}$ and $\mu_{L}$ values, (19) and (20) can be used to simulate the steady-state flow through the two CBVs and compare them with the measured flow in order to identify the four model parameters. As for the mechanical model, the parameter identification is carried out by means of numerical optimization. Also here the optimization routine is based on the fmincon function in MATLAB ${ }^{\circledR}$, which minimizes the squared deviation between the measured flow, $\widetilde{Q}_{C B V}$, and the simulated flow, $Q_{C B V}$ :

$$
\text { minimize } f=\left(\widetilde{Q}_{C B V}-Q_{C B V}\right)^{2}
$$

Fig. 16 show the measured and simulated flows after the parameter identification. The identified parameters are given in Table 8.

The steady-state simulation yields good conformity between the measured and simulated flows, which indicate that the suggested CBV model is valid and that the identified parameters are reliable.
Table 8: Model parameters for outer jib CBVs.

\begin{tabular}{llll}
\hline Parameter & Value & Parameter & Value \\
\hline$C_{1}$ & 1.6267 & $C_{2}$ & 0.2452 \\
$A_{0}$ & 27.84 & $A_{1}$ & 146.2 \\
$n_{0}$ & 0.4707 & $n_{1}$ & 0.0359 \\
\hline
\end{tabular}

For the actual verification of the model, a dynamic simulation is carried out with the joystick signal in Fig. 12 as input. The flow coefficients of the DCV are adjusted manually in order to simulate the correct flow and obtain the cylinder motion shown in Fig. 17.

Once the DCV model is calibrated and the simulated cylinder motion corresponds to the measured cylinder motion, the steady-state level of the simulated pressures can be compared with the measured pressures. Fig. 18 shows the simulated and measured values of $p_{2}$ and $p_{3}$.

There is indeed a good conformity between steadystate levels of the measured and simulated pressures, which verifies the steady-state characteristics of the CBV model and that the identified parameters are useful for simulation. 


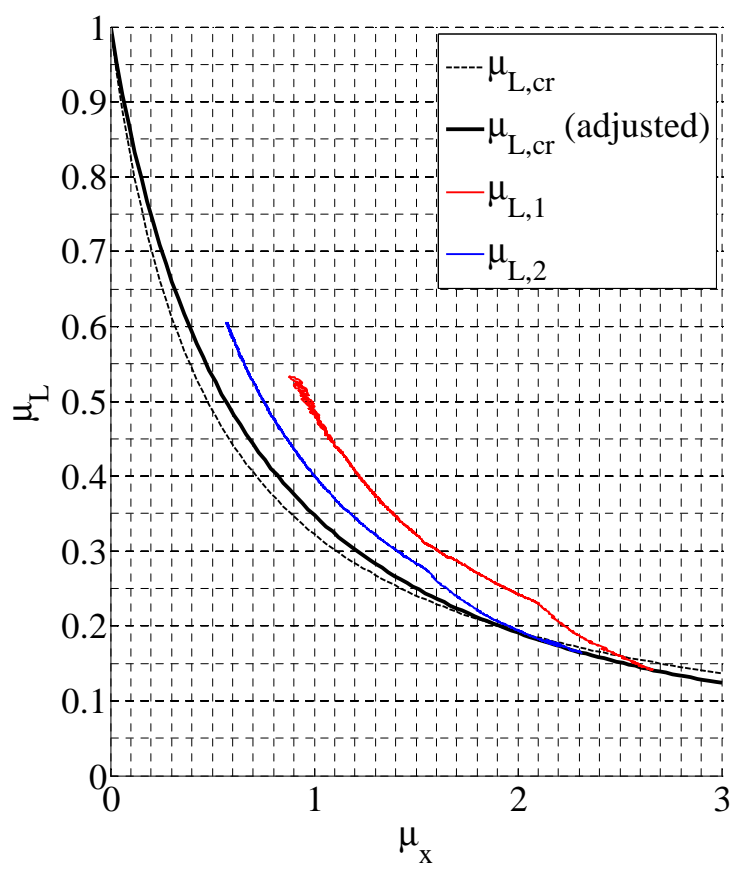

Figure 15: Relation between $\mu_{L}$ and $\mu_{x}$ for the outer jib CBVs.

\subsection{System Dynamics}

The remaining uncertain parameters that dominate the system dynamics are the flexibility and damping parameters of the mechanical system and the stiffness, bulk modulus, of the hydraulic oil. The actual friction in the hydraulic cylinders, the dynamics of hydraulic valves and other hydraulic components also influence the overall dynamics to some extent, but they are considered to be less important for the considered system. While the steady-state level of the simulated and measured pressures in Fig. 18 correspond well, there is a

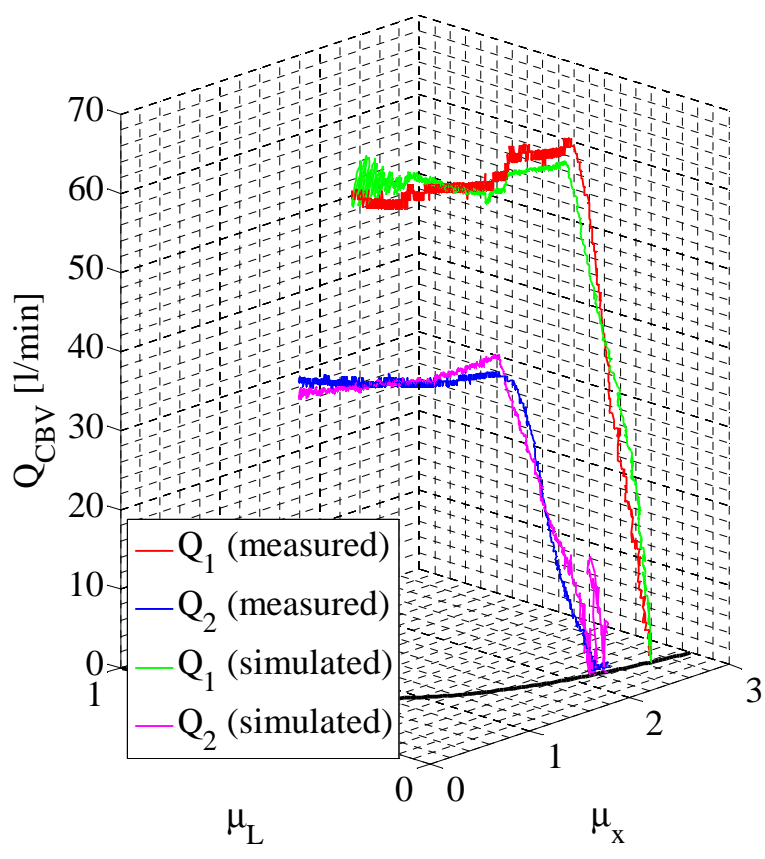

Figure 16: Measured and simulated flows through the CBVs after the parameter identification.

significant difference in dynamic response. The model is obviously stiffer than the real crane and therefore the flexibility parameters of the mechanical system are tuned by a scaling factor until the simulated dynamic response corresponds to the measured. Simultaneously, the bulk modulus is also adjusted.

The main objective for the tuning is to make the frequency of the simulated pressure oscillations correspond to the measured pressure oscillations. Naturally, the simulated amplitudes should also correspond to the measured amplitudes. However, there are more uncer-

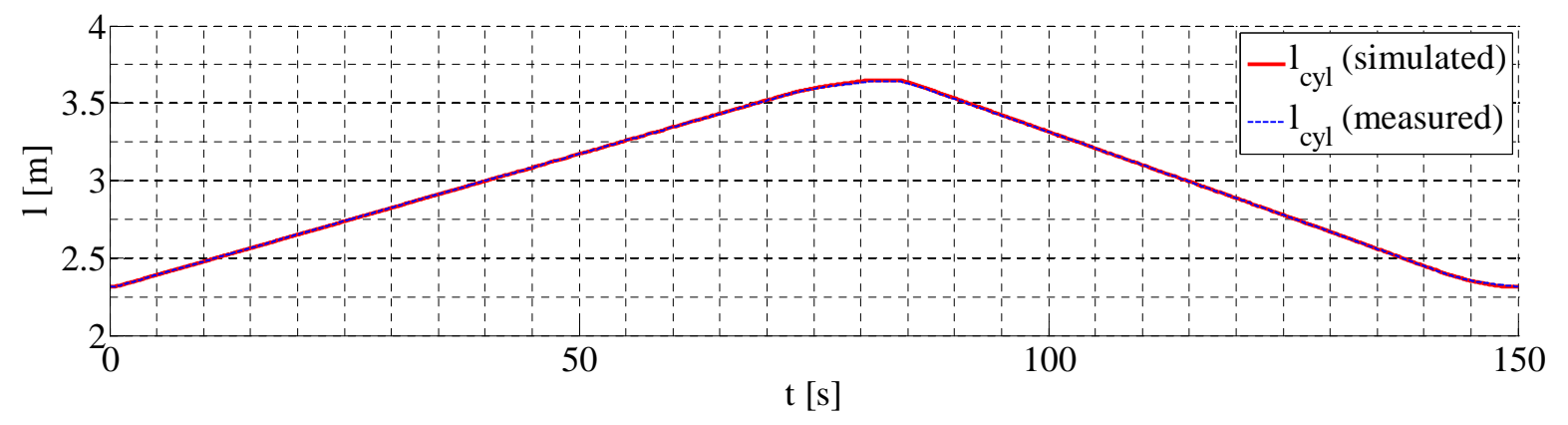

Figure 17: Measured and simulated cylinder motion. 


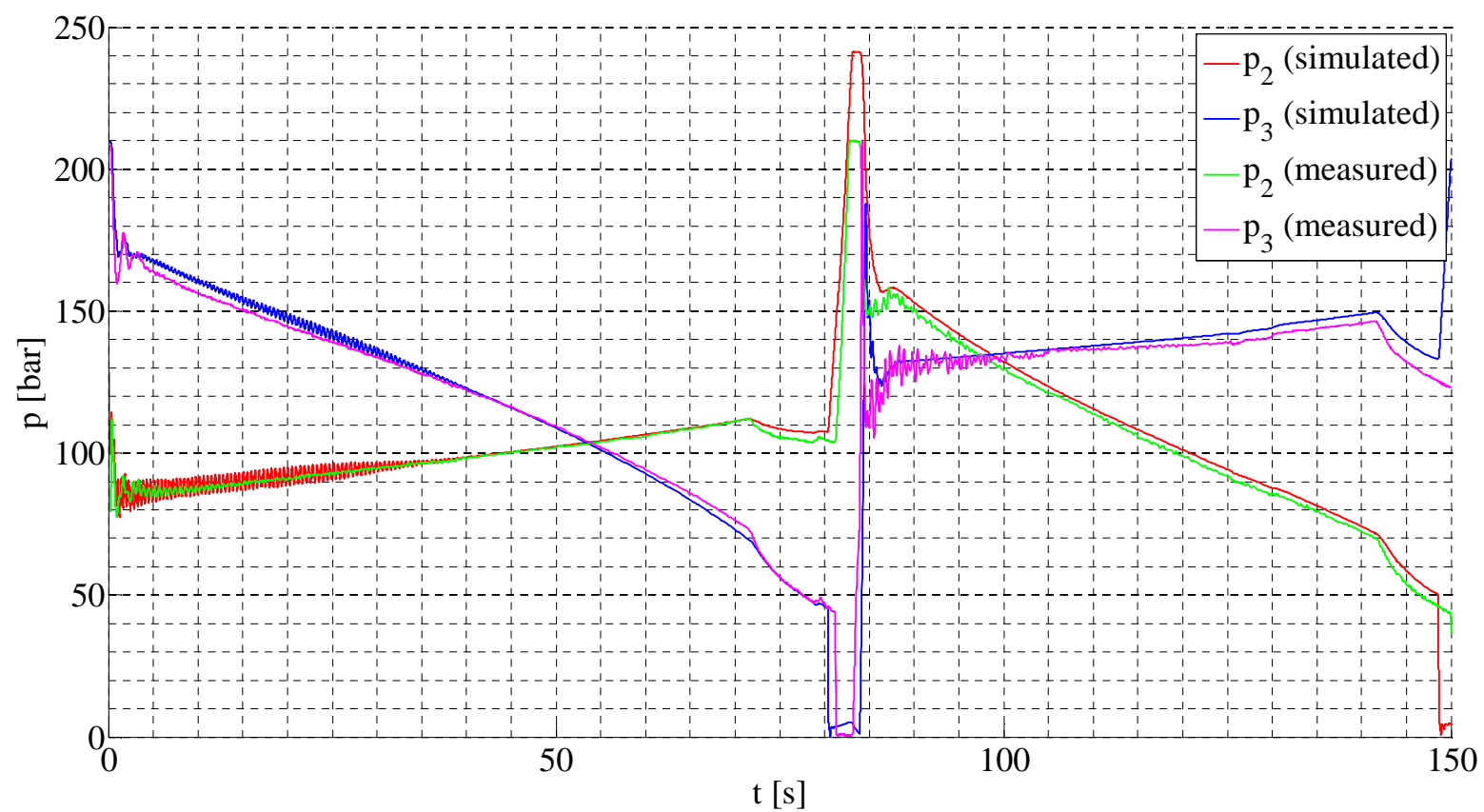

Figure 18: Measured and simulated pressures for verification of steady-state characteristics.

tain parameters related to the amplitude of the oscillations than for the frequency. Therefore some deviations are to be expected. Fig. 19 shows the simulated and measured pressures after the tuning.

During extension of the cylinder, both frequency and amplitude of the simulated pressure correspond very well with the measurements, except for the acceleration phase in the beginning of the sequence. The reason for this is most likely the un-modeled dynamics of the cylinder endstops and possibly that the CBV model is not accurate enough for accelerating flows.

During retraction of the cylinder only the frequency corresponds to some extent in the beginning of the sequence. The amplitudes do not correspond and the simulated oscillations are dampened far quicker than the measured oscillations. Also here, the likely causes are un-modeled dynamics of the cylinder endstop and inaccuracy of the CBV model. Furthermore, as described in section 4.3, the friction in the cylinder is quite complex around zero velocity and the applied cylinder model may be too simple to capture the real behavior during acceleration.

In general though, the simulated response corresponds well to the measurements. The observed deviations are within the expectations of what can be achieved with a model of the suggested detail level. The calibrated model is suitable for the type of simulations that can be utilized by system engineers working with hydraulic system design and/or control system design.

To obtain the correspondence shown in Fig. 19, all the flexibilty parameters are adjusted to $55 \%$ of their original value (Table 2) and the stiffness multipliers, $\beta_{k}$, used to determine the damping parameters for the three flexible members, are set to $0.002,0.003$ and 0.007 , respectively. Bulk modulus is set to 5500 bar, which is almost half the value of the initial guess.

Immediately, it seems surprising that the estimated structural stiffness needs to be reduced nearly by a factor of two. However, only three of the crane's structural components are considered flexible and while the estimated compliance of these individual members may be correct, the flexibility of the remaining components also contribute to the overall dynamics. These components include the king, the intermediate jib and the foundation on which the pedestal is mounted. In addition, the connections between the individual structural components may also offer some flexibility.

The identified value of bulk modules is significantly lower than the theoretical value of a typical hydraulic oil, even when accounting for a certain amount of entrained air. However, the compliance of pipes and hoses will also lower the effective bulk modulus. According to Merritt (1967) the effective bulk modulus is usually not more than 100,000 psi (approximately $6900 \mathrm{bar}$ ). The identified value of 5500 bar is therefore considered reliable. 


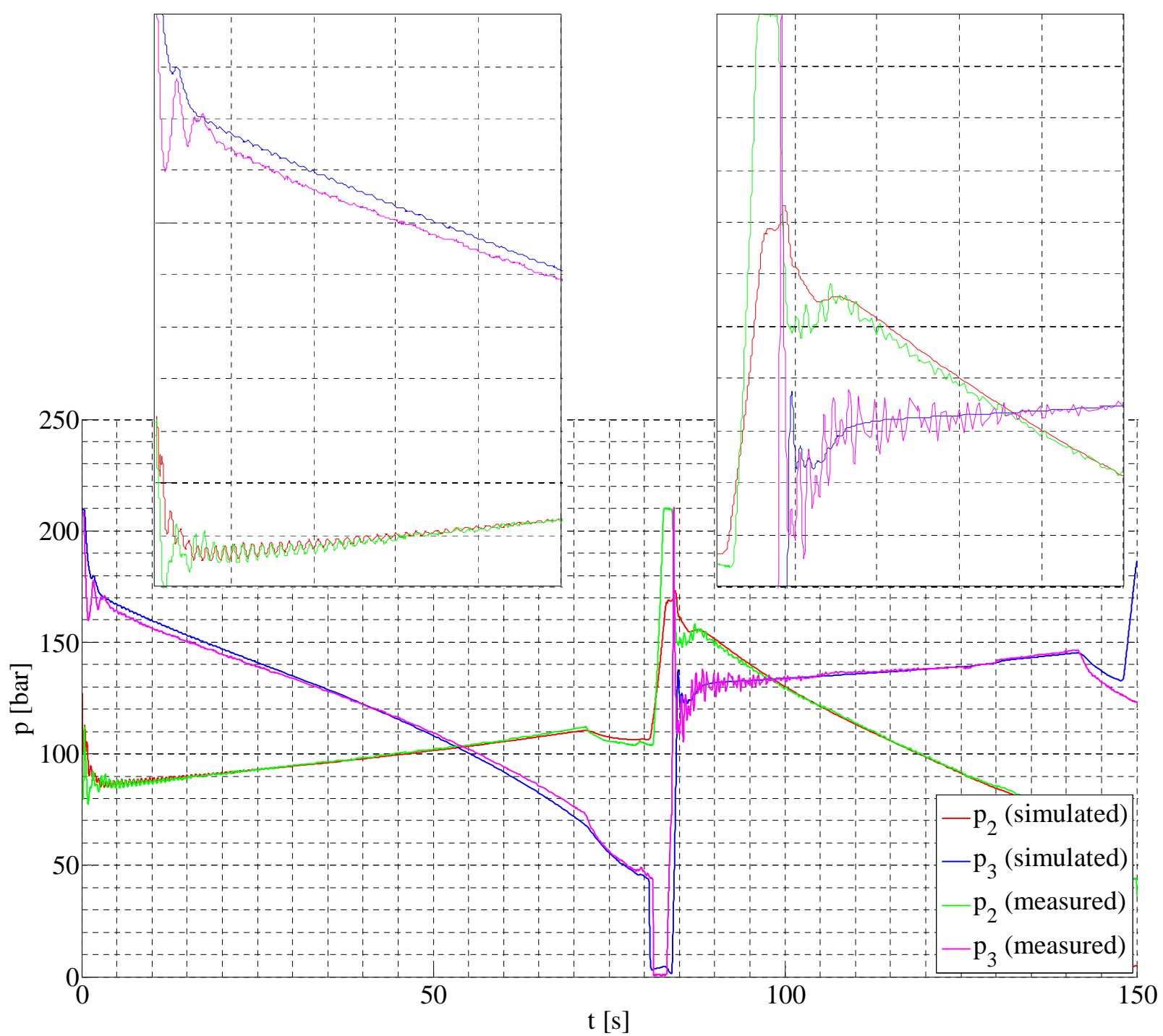

Figure 19: Measured and simulated pressures for verification of dynamic characteristics.

Estimating the damping of a complex system like the considered crane is obviously difficult. According to (5) the stiffness multipliers should be reduced along with the stiffness of the structural members, because the natural frequencies are lowered. Since the stiffness multipliers are actually increased, it implies that the damping ratio of $\zeta=0.03-0.07$ is too low. However, the un-modeled dynamics of structural components, other than the three considered, will also contribute to the total system damping. Furthermore the connections between the structural components will also offer some damping in terms of friction.

Therefore the stiffness multipliers determined in section 3.3 may be reasonable when considering the three structural members individually. For a complete model, though, the measurement show that additional damping needs to be introduced.

\section{Conclusions}

In this paper a model of an offshore knuckle boom crane has been presented. The model is developed in MapleSim ${ }^{\mathrm{TM}}$ and includes both the crane's mechanical system and the electro-hydraulic motion control system.

The mechanical system is modeled as a twodimensional multi-body system which includes the structural flexibility and damping. The finite segment method is used to model the flexibility and a procedure for estimating the structural damping is presented. Though these methods do not represent the 
state of the art within flexibility modeling, it is shown that they are sufficient for the given modeling purpose. Furthermore, they are advantageous in terms of modeling effort and computational requirements.

The motion control system is modeled using mostly semi-physical modeling techniques in order to reduce the computation requirements without neglecting or underestimating important physical phenomena. For modeling of the CBVs in the hydraulic system, a novel black-box approach is presented which uses two different pressure ratios to compute the flow through the valve. This approach is, however, based on having a certain amount of experimental data available.

The crane model is calibrated and verified with experimental data through three different steps:

1. Verfication of the steady-state characteristics of the mechanical system model by identifying the cylinder friction parameters and tuning the masses and COG positions of the bodies in the model.

2. Verification of the steady-state characteristics of the motion control system model, mainly by identifying the unknown CBV model parameters.

3. Verification of the dynamic behavior of the crane model by tuning the flexibility and damping parameters of the mechanical system model and the stiffness, bulk modulus, of the hydraulic oil.

For the first two steps an optimization procedure is applied to efficiently identify the unknown parameters. In the last step the estimated parameters are simply adjusted by a scaling factor until the simulated response corresponds to the measured response.

The demonstrated modeling and parameter identification techniques target the system engineer by taking into account the limited access to component data normally encountered by engineers working with system design. The verified crane model is an example of a virtual prototype which can be used to evaluate and improve the design of the considered system.

\section{Acknowledgements}

The work presented in this paper is funded by the Norwegian Ministry of Education and Research and Aker Solutions.

The authors would like to thank Morten Abusdal (Aker Solutions) for his help with the experimental work.

\section{References}

Abdel-Rahman, E. M., Nayfeh, A. H., and Masoud, Z. N. Dynamics and control of cranes: A review.
Journal of Vibration and Control, 2003. 67(7):863908. doi:10.1177/1077546303009007007.

Adams, V. and Askenazi, A. Building better products with finite element analysis. OnWord Press, 1999.

Bak, M. K. and Hansen, M. R. Modeling, performance testing and parameter identification of pressure compensated proportional directional control valves. In Proceedings of the 7th FPNI PhD Symposium on Fluid Power. Reggio Emilia, Italy, pages 889-908, 2012.

Bak, M. K., Hansen, M. R., and Nordhammer, P. A. Virtual prototyping - model of offshore knuckle boom crane. In Proceedings of the 24th International Congress on Condition Monitoring and Diagnostics Engineering Management. Stavanger, Norway, pages 1242-1252, 2011.

Connelly, J. and Huston, R. L. The dynamics of flexible multibody systems: A finite segment approach - I. Theoretical aspects. Computers and Structures, 1994a. 50(2):255-258. doi:10.1016/00457949(94)90300-X.

Connelly, J. and Huston, R. L. The dynamics of flexible multibody systems: A finite segment approach - II. Example problems. Computers and Structures, 1994b. 50(2):259-262. doi:10.1016/00457949(94)90301-8.

Ellman, A., Käppi, T., and Vilenius, M. J. Simulation and analysis of hydraulically driven boom mechanisms. In Proceedings of the 9th Bath International Fluid Power Workshop. Bath, UK, pages 413-429, 1996.

Esque, S., Käppi, T., and Ellman, A. Importance of the mechanical flexibility on behaviour of a hydraulic driven log crane. In Proceedings of the 2nd International Conference on Recent Advances in Mechatronics. Istanbul, Turkey, pages 359-365, 1999.

Esque, S., Raneda, A., and Ellman, A. Techniques for studying a mobile hydraulic crane in virtual reality. International Journal of Fluid Power, 2003. 4(2):2534.

Hansen, M. R., Andersen, T., and Conrad, F. Experimentally based analysis and synthesis of hydraulically actuated loader crane. In Bath Workshop on Power Transmission and Motion Control. Bath, UK, pages 259-274, 2001.

Henriksen, J., Bak, M. K., and Hansen, M. R. A method for finite element based modeling of flexible components in time domain simulation of knuckle 
boom crane. In Proceedings of the 24th International Congress on Condition Monitoring and Diagnostics Engineering Management. Stavanger, Norway, pages 1215-1224, 2011.

Hiller, M. Modelling, simulation and control design for large and heavy manipulators. Robotics and Autonomous Systems, 1996. 19(2):167-177. doi:10.1016/S0921-8890(96)00044-9.

Huston, R. L. Multi-body dynamics including the effects of flexibility and compliance. Computers and Structures, 1981. 14(5-6):443-451. doi:10.1016/00457949(81)90064-X.

Huston, R. L. Computer methods in flexible multibody dynamics. International Journal for Numerical Methods in Engineering, 1991. 32(8):1657-1668. doi:10.1002/nme.1620320809.

Huston, R. L. and Wang, Y. Flexibility effects in multibody systems. In Computer Aided Analysis of Rigid and Flexible Mechanical Systems: Proceedings of the NATO Advanced Study Institute, pages 351376. Troia, Portugal, 1993.

Merritt, H. E. Hydraulic control systems. Wiley, 1967.

Mikkola, A. and Handroos, H. Modeling and simulation of a flexible hydraulic-driven log crane. In Proceedings of the 9th Bath International Fluid Power Workshop. Bath, UK, 1996.
Mostofi, A. The incorporation of damping in lumpedparameter modelling techniques. Proceedings of the Institution of Mechanical Engineers, Part K: Journal of Multi-body Dynamics, 1999. 213(1):11-17.

Nielsen, B., Pedersen, H. C., Andersen, T. O., and Hansen, M. R. Modelling and simulation of mobile hydraulic crane with telescopic arm. 2003. pages 433-446.

Nikravesh, P. E. Computer-aided analysis of mechanical systems. Prentice Hall, 1988.

Ottestad, M., Nilsen, N., and Hansen, M. R. Reducing the static friction in hydraulic cylinders by maintaining relative velocity between piston and cylinder. In Proceedings of the 12th International Conference on Control, Automation and Systems. Jeju Island, Korea, pages 764-769, 2012.

Shabana, A. A. Flexible multibody dynamics: Review of past and recent developments. Multibody System Dynamics, 1997. 1(2):189-222. doi:10.1023/A:1009773505418.

Than, T. K., Langen, I., and Birkeland, O. Modelling and simulation of offshore crane operations on a floating production vessel. In Proceedings of The Twelfth (2002) International Offshore and Polar Engineering Conference. Kitakyushu, Japan, 2002. 